showed that working conditions substantially changed (i.e. difference of one standard deviation) between two waves. Social support and emotional job demands had the highest amounts of substantial changes (17\% and 19\%), while physical demands remained relatively stable ( $6 \%$ substantial change). After the first two waves, about $12 \%$ of workers with a chronic illness left paid employment. Results of discrete-time survival models are expected to be available in 2019 (by the time of the EPICOH conference).

Conclusion Ensuring that working conditions can be adapted to the needs of older workers who have a chronic disease may help to extend working life.

\section{P.2.31 ASSOCIATION BETWEEN WORK AND CHRONIC OBSTRUCTIVE PULMONARY DISEASE REVIEW OF SYSTEMATIC REVIEWS}

Henk van der Molen*, Gerda de Groene, Carel Hulshof, Monique Frings-Dresen. Amsterdam UMC, Coronel Institute of Occupational Health, Netherlands Center for Occupational Diseases, Amsterdam Public Health research institute, Amsterdam, Netherlands

\subsection{6/OEM-2019-EPI.259}

Introduction Chronic Obstructive Pulmonary Disease (COPD) is a highly prevalent disease with reported prevalence up to $12 \%$. Although smoking is one of the main factors in causing COPD, evidence from systematic reviews grows that also occupational exposures contribute. To examine whether work-related risk factors are associated with chronic pulmonary obstructive disease (COPD) a review of systematic reviews was performed.

Methods Medline was searched from 2009 until 20 June 2017 for systematic reviews. Systematic reviews were included when outcome data were described in terms of clinically assessed COPD by means of data on lung function and at least two levels of work-related exposure were mentioned (exposed versus low or non-exposed). One author selected studies and extracted data, two authors assessed study quality with AMSTAR.

Result Eight systematic reviews met the inclusion criteria. In all reviews various exposures to vapour, dusts, gases, and fumes (VGDF) at work are associated with COPD. Most of the included studies are cross-sectional and show a high heterogeneity in population, setting and - mostly self-reported exposures. Two high-quality reviews including meta-analyses show associations and excess risk of COPD for work-related general exposure to VGDF with a summary odds ratio of 1.4 (95\% CI 1.19-1.73) and for work exposure to inorganic dust with a mean difference in predicted FEV1 of $-5.7 \%$ (95\%CI: - $8.62 \%$ to $-2.71 \%)$.

Discussion Exposure to VGDF at work is associated with a small but increased risk of COPD. More detailed workplace measurements of specific VGDF are warranted to gain an insight into dose-response relationships.

\section{P.2.32 CHANGE IN EMPLOYMENT STATUS IN SURVIVORS WITH OCCUPATIONAL CANCERS}

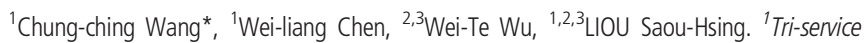
General Hospital, National Defense Medical Center, Taipei, Taiwan; ${ }^{2}$ Department of Public Health, National Defense Medical Center, Taipei, Taiwan, Republic of China., Taipei, Taiwan; ${ }^{3}$ National Institute of Environmental Health Sciences, National Health Research Institutes, Miaoli, Taiwan

10.1136/OEM-2019-EPI.260
Background The aim of this study is to investigate the sociodemographic information and employment status for 2 years after diagnosis with occupational cancers.

Method In the Labor Registry Cohort of Taiwan during the period 2004-2015, 86 workers diagnosed with occupational cancers were identified. The cancer group included all kind of cancer diagnosed during this period. The cancer diagnoses were recorded according to the International Classification of Diseases for Oncology. Sex, age, industrial classifications, and occupational status were registered for the year 2004. The person's annual wage and salary income was registered in New Taiwan Dollar (NTD) and divided into tertiles of 16500-22800, 24000-40100, and 4200043900.

Results There were 86 eligible workers with occupational cancers in our cohort study. All eligible participants were categorized into 2 groups based on their work status. There were 61 unemployed survivors with mean age of 53.05 \pm 8.3 and 25 employed survivors with mean age of 46.2 \pm 9.2 (table 1). Employed survivors had younger age and lower salary income than unemployed survivors. At 2 year of follow-up (figure 1), there was substantial declined trend for employment percentage (from $100 \%$ to 29\%). For change of salary income, increased percentage of salary income change was observed. In different age groups (figure 2), young group aged 28-45 years had significantly higher employment percentages than older groups aged 46-55 years and 56-71 years. Notably, there were elevated percentage of salary income change in older group aged 56-71 years.

Conclusion Our study highlighted that the workers with occupational cancers had apparent dilemmas in the workplace 2 years after diagnosis.

\section{P.2.33 THE ASSOCIATION BETWEEN PATTERN OF SHIFT WORK AND SLEEP QUALITY AMONGST NURSES IN UNIVERSITY HOSPITAL IN NORTHEASTERN REGION OF THAILAND}

Kampanat Wangsan*, Naesinee Chaiear, Kittisak Sawanyawisuth, Paanumas Krisorn. Faculty of Medicine, Khon Kaen University, Muang, Thailand

\subsection{6/OEM-2019-EPI.261}

Introduction Shift work associated with many health problems, work performance and injury. Disturbance of sleep quality is the main mechanism of shift work effects. Nurses are at risk of many occupational hazards and also shift work. The information about shiftwork and the association of sleep quality amongst nurses of Thailand is still limit.

Objective To study the association of shift work and quality of sleep and which pattern has highest risk of poor quality of sleep amongst nurses in university hospital in Northeastern region of Thailand.

Material and method One thousand and one hundred nurses were met inclusion and exclusion criteria with $75.5 \%(n=831)$ participation rate. The data was collected via questionnaire including personal information, shift schedule, depression screening questionnaire, obstructive sleep apnea screening questionnaire and sleep quality questionnaire (PSQI). Crosssectional analytical study was conducted.

Result All patterns of shift work amongst sample were irregular shift work. There were statistically significant association between shift work and poor quality of sleep. Predominant backward shift pattern has higher association 